\title{
Increased levels of HMGB1 in trophoblastic debris may contribute to preeclampsia
}

\author{
Jun Shao ${ }^{1,2}$, Mingzhi Zhao ${ }^{1,2}$, Mancy Tong ${ }^{2}$, Jia Wei ${ }^{2}$, Michelle R Wise ${ }^{2}$, Peter Stone ${ }^{2}$, \\ Lawrence Chamley ${ }^{2}$ and Qi Chen ${ }^{1,2}$ \\ ${ }^{1}$ The Hospital of Obstetrics \& Gynaecology, Fudan University, China and ${ }^{2}$ Department of Obstetrics \& Gynaecology, \\ The University of Auckland, New Zealand \\ Correspondence should be addressed to Q Chen or M Zhao; Email: q.chen@auckland.ac.nz or 34996817@qq.com
}

\begin{abstract}
Preeclampsia is triggered by an as yet unknown toxin from the placenta. Antiphospholipid antibodies (aPL), a strong risk factor for preeclampsia, have been shown to induce the production of toxic trophoblastic debris from the placenta. High mobility group box 1 (HMGB1) is a proinflammatory danger signal, and the expression of it has been reported to be increased in preeclampsia. This study examined whether aPL or preeclamptic sera increase the expression of HMGB1 in the syncytiotrophoblast or trophoblastic debris. Trophoblastic debris from normal placental explants that had been cultured with aPL or preeclamptic sera was exposed to endothelial cells. Endothelial cell activation was quantified by cell-surface ICAM-1 expression and U937 monocyte adhesion. The expression of HMGB1 in placental explants and trophoblastic debris that had been treated with aPL or preeclamptic sera was measured by immunohistochemistry and western blotting. The expression of the receptor for advanced glycation end products (RAGE) in endothelial cells was quantified by western blotting. Compared with controls, the expression of HMGB1 in the cytoplasm of the syncytiotrophoblast and trophoblastic debris was increased by treating placental explants with aPL or preeclamptic sera. The increased levels of HMGB1 contributed to endothelial cell activation, mediated in part by the RAGE. Preeclamptic sera and aPL both induced an increase in the cytoplasmic levels of the danger signal HMGB1 in trophoblastic debris. This increased HMGB1 in trophoblastic debris may be one of the toxic factors released from the placenta in preeclampsia.

Reproduction (2016) 152 775-784
\end{abstract}

\section{Introduction}

Preeclampsia is a human pregnancy-specific disease, characterised by multisystem dysfunction. It is generally manifested by maternal hypertension after 20 weeks of gestation, and it is a leading cause of maternal and perinatal mortality and morbidity (Sibai et al. 2005). Before the onset of symptoms, women destined to develop preeclampsia exhibit endothelial cell activation and an exaggerated inflammatory response. Although the complete pathogenesis of preeclampsia is still uncertain, it seems that preeclampsia is triggered by a placental factor(s), given that preeclampsia resolves after delivery. A number of studies have reported that the inflammatory cytokines are increased in preeclampsia at presentation (Lau et al. 2013), suggesting that a mediator of inflammation is involved in the pathogenesis of preeclampsia (Redman \& Sargent 2010).

During pregnancy, a large amount of trophoblastic debris is shed from syncytiotrophoblast into maternal circulation by an apoptotic process as early as six weeks of gestation (Covone et al. 1984). However, in preeclampsia, there is a well-documented increase in the amount of trophoblast debris shed into the maternal blood (Douglas et al. 1959, Attwood \& Park 1961, Knight et al. 1998). In addition, a switch from the shedding of apoptotic (safe) to necrotic (dangerous/ toxic) trophoblastic debris may be one of the placental triggers of preeclampsia (Huppertz et al. 2003).

The intracellular protein high mobility group box 1 (HMGB1) was originally described as a DNA-binding nuclear protein and transcription factor, but more recently, has been shown to be a proinflammatory danger signal that causes sterile inflammation when released from necrotic cells or cells under stress (Lotze \& Tracey 2005). HMGB1 is expressed by trophoblasts (Wang et al. 2011) and can be found in either the nucleus or the cytoplasm of these cells. In general, when HMGB1 is translocated to the cytoplasm or released to the extracellular environment, it acts as a danger signal (Tsung et al. 2014). Circulating levels of HMGB1 are increased in many inflammation-related diseases including preeclampsia (Tsung et al. 2014). We have recently shown that the expression of HMGB1 was increased in syncytiotrophoblast in the placenta of women with preeclampsia and was associated with the severity of preeclampsia (Chen et al. 2016b). 
Once released from necrotic cells, HMGB1 can interact with the receptor for advanced glycation end products (RAGE) (Hori et al. 1995) or toll-like receptor (TLR) 2 or TLR4 (Park et al. 2006, Bianchi \& Manfredi 2007, Campana et al. 2008), and HMGB1 released from cells into the extracellular environment may play an important role in the response to tissue damage and particularly necrotic cell death.

An association between antiphospholipid antibodies (aPL) and preeclampsia is well documented (Noris et al. 2005). Treating normal placental explants with aPL increases the amount of dangerous/necrotic trophoblastic debris shed from the explants, and this necrotic trophoblastic debris activates the endothelial cells (Duckitt \& Harrington 2005, Chen et al. 2009b). Sera from women with preeclampsia have also been shown to induce the production of dangerous/necrotic trophoblastic debris (Chen et al. 2012b, 2013, Shen et al. 2014). However, whether there is an association between aPL or sera from preeclampsia and increased levels of HMGB1 in trophoblastic debris has not been fully investigated. Therefore, we undertook this study to investigate the role of HMGB1 in dangerous trophoblast debris from aPL-treated placentae in inducing endothelial cell activation. Our secondary objective was to examine the expression of RAGE, one of the HMGB1 receptor in endothelial cells.

\section{Materials and methods}

This investigation conforms to the principles outlined in the Declaration of Helsinki. This study was approved by the Auckland Regional Health and Disabilities Ethics Committee, New Zealand. All patient-derived tissue and blood samples were obtained with written informed consent.

\section{Antiphospholipid antibodies (aPL)}

The murine monoclonal antiphospholipid antibody, ID2 (Chamley et al. 2001), was produced in this laboratory and generated by hybridoma culture, and then purified using HiTrap Protein G columns (GE Healthcare). This monoclonal aPL has been extensively characterised and has, anticardiolipin, anti $\beta_{2} \mathrm{GPI}$ and lupus anticoagulant activities (Pantham et al. 2015). A murine IgG1 antibody (Life Technologies) was used as an isotype-matched treatment control antibody in experiments involving ID2.

\section{Collection of placentae and blood}

Fifteen first-trimester placentae were collected after elective surgical terminations of on-going pregnancies ranging from 8 to 12 weeks of gestation. In addition, blood samples from six women with preeclampsia and six gestation-matched normotensive pregnant women were collected by venepuncture into plain vacutainer tubes. The blood was allowed to clot, centrifuged at $2500 \mathrm{~g}$ and the serum was aspirated and stored in aliquots at $-80^{\circ} \mathrm{C}$.
Preeclampsia was defined as maternal systolic blood pressure $\geq 140 \mathrm{mmHg}$ and/or diastolic blood pressure $\geq 90 \mathrm{mmHg}$ on two occasions separated by $6 \mathrm{~h}$, and proteinuria $>300 \mathrm{mg}$ in a 24-h period after 20 weeks of gestation following the guidelines of the American College of Obstetricians and Gynaecologists ('Hypertension in Pregnancy' 2013).

\section{Cell culture}

All cell culture reagents including culture media, foetal bovine serum (FBS) and Cell Tracker, CMTPX (Red) were purchased from Invitrogen. The human microvascular endothelial cell line (HMEC-1) originally derived from dermal microvascular endothelial cells (Ades et al. 1992) was obtained from the National Centre for Infectious Diseases (USA) and grown to confluence in MCDB 131 media as described previously (Chen et al. 2006). The endothelial cells were seeded at a density of $10^{4}$ cells in each well of 96-well sterile culture plates or the equivalent density $\left(8 \times 10^{4}\right)$ in the wells of a 12 -well culture plate and grown to confluence. The passages of HMEC-1 used in this study were under 15. U937 cells, a human monocyte cell line, were grown in DMEM/F12 media supplemented with $10 \%$ of FBS.

\section{Culture of placental explants and preparation of trophoblastic debris}

Trophoblastic debris was collected from first-trimester placentae $(n=15)$ that had been treated with the aPL ID2 $(20 \mu \mathrm{g} / \mathrm{mL})$ or isotype-matched control antibody $(20 \mu \mathrm{g} / \mathrm{mL})$ or sera, from women with preeclampsia or normotensive controls, as described previously (Abumaree et al. 2006, Chen et al. 2006). Briefly, approximately $400 \mathrm{mg}$ explants were dissected from first-trimester placentae. The explants were then cultured in Netwell culture inserts, suspended in 12-well culture plates, for $24 \mathrm{~h}$ at $37^{\circ} \mathrm{C}$ in DMEM/F12-containing $10 \%$ FBS in an ambient oxygen atmosphere containing $5 \% \mathrm{CO}_{2}$ in the presence of aPL $(20 \mu \mathrm{g} / \mathrm{mL})$ or isotype-matched control antibody $(20 \mu \mathrm{g} / \mathrm{mL})$. In other experiments, the explants were treated with $10 \%$ serum from each of the six women with preeclampsia or serum from each of six normotensive women, and in addition, fifteen untreated, control explants were cultured with medium without human serum, for $24 \mathrm{~h}$. The Netwell inserts (containing the explants) were then removed from the culture wells and the trophoblastic debris shed from the explants, which passes through the Netwell inserts, was aspirated from the culture wells and centrifuged at $300 \mathrm{~g}$ for $10 \mathrm{~min}$. The supernatant was discarded and washed with PBS, and the trophoblastic debris was resuspended in PBS buffer, and then depleted of contaminating $\mathrm{CD} 45^{+}$leukocytes using magnetic beads (Dynal, Invitrogen) according to the manufacturer's instructions. Contaminating red blood cells were removed by incubation in MilliQ water for one minute, and then one volume of $10 \times$ PBS was added to immediately return the trophoblastic debris to isotonic conditions. These procedures resulted in trophoblastic debris (confirmed by cytokeratin 7 and vimentin immunostaining) essentially free from contaminating cells as described previously (Abumaree et al. 2006, Chen et al. 2009a, 2010). The trophoblastic debris 
from approximately $1200 \mathrm{mg}$ of placental explants was pooled and added to endothelial cell cultures.

\section{Immunohistochemistry}

The expression levels of HMGB1 in trophoblastic debris or first-trimester placentae that had been treated with $\mathrm{aPL}$ or an isotype-matched control antibody, or preeclamptic or normotensive pregnant sera were measured by immunohistochemistry. Non-specific antibody binding was blocked by incubating with $10 \%$ normal goat serum in PBS for $10 \mathrm{~min}$ at room temperature. Murine anti-human HMGB1 monoclonal antibody (diluted 1:100 in 10\% normal goat serum, Abcam, Sapphire Biosciences, Sydney) was added for one hour at room temperature. Sections were then washed with PBST and incubated with $10 \% \mathrm{H}_{2} \mathrm{O}_{2}$ in methanol for $3 \mathrm{~min}$. After washing three times with goat anti-mouse IgG, biotinconjugated antibody (Jackson Immunoresearch Laboratories) was then added for one hour at room temperature. After washing, streptavidin-conjugated horse radish peroxidase (HRP) was added for a further hour. After washing, the sections were stained for $15 \mathrm{~min}$ with 3-amino-9-ethylcarbazole (AEC) using a kit according to the manufacturers instruction (Dako, Global Science), and sections were then counter-stained for three minutes with haematoxylin. Negative staining controls were performed as mentioned previously but omitting the primary (anti-HMEC1) antibody.

\section{Determination of endothelial cell activation by cell-surface ICAM-1 ELISA}

HMEC-1 endothelial cells were grown until confluent in 96-well culture plates in MCDB 131 media. The cell cultures were incubated with trophoblastic debris from aPL-treated, isotypematched control antibody-treated or -untreated placental explants or with human recombinant HMGB1 $(0.2 \mu \mathrm{g} / \mathrm{mL}$ and $2 \mu \mathrm{g} / \mathrm{mL}$ ) for $24 \mathrm{~h}$. In some experiments, neutralising RAGE antibody was added to the endothelial cell cultures for $60 \mathrm{~min}$ before adding the HMGB1. The neutralising antibody was maintained in the cultures along with the HMGB1. After 24h, remaining trophoblastic debris or HMGB1 was washed out from the cultures, and the cell-surface expression of ICAM-1 by the HMEC-1 monolayers was determined by cell-based ELISA as described previously (Chen et al. 2006).

\section{Determination of endothelial cell activation by monocyte adhesion assay}

HMEC-1 endothelial cells were grown and exposed to trophoblastic debris or recombinant HMGB1 as for the ICAM-1 ELISAs mentioned previously. After exposure to trophoblastic debris or HMGB1 for $24 \mathrm{~h}$, the remaining HMGB1 or trophoblastic debris was washed off, and then the U937 cells that had been labelled with cell tracker, CMTPX (Red) were added, and the incubation was continued for three hours at $37^{\circ} \mathrm{C}$. The cultures were then washed to remove nonadherent U937 cells, the adherent U937 cells were quantified using a fluorescence plate reader (Synergie 2, BioTek). Data were expressed as the fold increase of adhesion of U937 cells relative to the control, untreated endothelial cells.

\section{Determination of the expression of HMGB1 in trophoblastic debris by western blotting}

The relative levels of HMGB1 in trophoblastic debris from first-trimester placental explants that had been treated with aPL or isotype-matched control antibody were measured by western blotting. Trophoblastic debris was homogenised in RIPA buffer $(50 \mathrm{mM}$ Tris, $150 \mathrm{mM} \mathrm{NaCl}, 1 \%$ sodium deoxycholate, $0.1 \%$ SDS, $1 \%$ Nonidet P40 substitute, protease inhibitor, $1 \mathrm{mM}$ phenylmethanesulfonylfluoride). All samples $(20 \mu \mathrm{g}$ of total protein) were loaded on $14 \%$ SDS-PAGE gels and electrophoresed, and then transferred to nitrocellulose membranes. Non-specific binding was blocked by incubating membranes in the blocking solution (10\% non-fat milk in PBST) for $1 \mathrm{~h}$ at room temperature, and then membranes were incubated with mouse anti-human HMGB1 antibody (1:250) in blocking solution for $2 \mathrm{~h}$ at room temperature. After washing with PBST three times, the membranes were incubated with HRP-conjugated goat anti-mouse antibody (1:2000, Jackson Immunoresearch Laboratories, Pennsylvania) for $1 \mathrm{~h}$ at room temperature. After washing with PBST, the membranes were incubated with Amersham ECL Prime Western blotting detection reagent. Chemiluminescence from the membranes was detected using an Image Quant LAS3000 (Thermo Fisher). Protein levels of HMGB1 were analysed relative to the $\beta$-actin loading control. $\beta$-Actin was detected on the membranes as follows. Membranes were stripped with stripping buffer $(62.5 \mathrm{mM}$ Tris- $\mathrm{Hcl}$, $\mathrm{pH} 6.7,2 \% \mathrm{SDS}$ and $100 \mathrm{mM} 2 \mathrm{ME}$ ) at $50^{\circ} \mathrm{C}$ for $30 \mathrm{~min}$, and then non-specific binding was blocked by incubation in blocking solution for one hour. Then the membranes were incubated with mouse anti-human $\beta$-actin (1:5000, Abcam, Sapphire Biosciences, Sydney) for $2 \mathrm{~h}$ at room temperature. After washing three times with PBST, the membranes were incubated with HRP-conjugated goat anti-mouse antibody (1:5000, Jackson Immunoresearch Laboratories, Pennsylvania) for $1 \mathrm{~h}$ at room temperature. After washing with PBST, the membranes were incubated with AmershamTM ECLTM Prime Western blotting detection reagent. Chemiluminescence from the membranes was detected using an Image Quant LAS3000 (Thermo Fisher).

\section{Determination of the expression of $H M G B 1$ in the cytoplasm of placental explants by western blotting}

Placental explants were treated with aPL or isotype-matched control antibody $(n=4)$ or preeclamptic or normotensive sera $(n=6)$. The explants were washed three times with PBS, and then incubated in $500 \mu \mathrm{L}$ RIPA buffer on ice for $6 \mathrm{~h}$ or $24 \mathrm{~h}$ without agitation. Samples were then centrifuged at $13,000 \mathrm{~g}$ for $10 \mathrm{~min}$, and the supernatants were collected for western blotting. All samples $(20 \mu \mathrm{g}$ of total protein) were loaded on SDS-PAGE gels and electrophoresed, and then transferred to nitrocellulose membranes, which were probed for HMGB1 as mentioned previously. After detecting HMGB1, the membranes were washed extensively and incubated with rabbit anti-human Lamin B1 antibody (1:750, Abcam, Sapphire Biosciences, Sydney) in blocking solution for $2 \mathrm{~h}$ at room temperature. After washing with PBST three 
times, the membranes were incubated with HRP-conjugated goat anti-rabbit antibody (1:2000, Jackson Immunoresearch Laboratories, Pennsylvania) for $1 \mathrm{~h}$ at room temperature. After washing with PBST, the membranes were incubated with Amersham ECL Prime Western blotting detection reagent. Chemiluminescence from the membranes was detected using an Image Quant LAS3000 (Thermo Fisher).

\section{Determination of the expression of RAGE in endothelial cells by western blotting}

The relative levels of RAGE in endothelial cells that had been treated with trophoblastic debris shed from first-trimester placental explants that had been treated with aPL or isotypematched-control antibody or sera from preeclamptic or normotensive pregnant women were measured by western blotting. Endothelial cells were homogenised in RIPA buffer. All samples $(20 \mu \mathrm{g})$ were loaded on $14 \%$ SDS-PAGE gels and electrophoresed, and then transferred to nitrocellulose membranes. Non-specific binding was blocked by incubating membranes in blocking solution for $1 \mathrm{~h}$, and then membranes were incubated with mouse anti-human RAGE antibody (1:500, Sigma) in blocking solution for $2 \mathrm{~h}$ at room temperature. After washing with PBST, the membranes were incubated with biotin-conjugated goat anti-mouse antibody $(1: 2000)$ for $1 \mathrm{~h}$ at room temperature. After washing with PBST, the membranes were then incubated with streptavidinconjugated HRP (1:3000, Jackson Immunoresearch Laboratories, Pennsylvania) for $1 \mathrm{~h}$ at room temperature. After washing with PBST, the membranes were incubated with Amersham ECL Prime Western blotting detection reagent. Chemiluminescence from the membranes was detected using an Image Quant LAS3000 (Thermo Fisher). Protein levels of RAGE were analysed relative to the $\beta$-actin loading control as mentioned previously.

\section{Determination of soluble levels of HMGB1 in conditioned medium}

The levels of HMGB1 in conditioned medium from placental explants that had been treated with $\mathrm{aPL}$, isotype-matched control antibody or sera from preeclamptic or normotensive pregnant women were measured by ELISA according to the manufacturer's instructions (Lifespan BioSciences, Seattle, WA, USA).

\section{Statistical analysis}

Data for measuring ICAM-1 expression by endothelial cells and $U 937$ adhesion to endothelial cells were conducted in quadruplicate and repeated at least three times. Data are presented as the median and 5th and 95th percentiles of the fold change relative to untreated controls. Data for the levels of soluble HMGB1 in conditioned medium are presented as the mean and S.D. The statistical significance of the results was assessed by a Mann-Whitney $U$ test using the Prism software package. Semi-quantification of western blotting was analysed by a Mann-Whitney $U$ test using Prism software package. $P<0.05$ was considered as statistically significant.

\section{Results}

\section{The expression of HMGB1 was increased by treating placental explants with aPL}

To investigate whether aPL changed the expression of HMGB1, first-trimester placental explants were treated with aPL or an isotype-matched control antibody, and the expression of HMGB1 was measured in both trophoblastic debris and the placental explants by immunohistochemistry. In control-antibody-treated or -untreated explants, the expression of HMGB1 was limited predominantly to the nuclei of cells of the mesenchymal core with rare staining of nuclei in the syncytiotrophoblast (Fig. 1A: untreated and B: isotype matched control antibody treated). There was very low cytoplasmic staining for HMGB1 in any cell type in control explants. In explants treated with aPL, the nuclear staining for HMGB1 in mesenchymal cells was retained, but there was strong cytoplasmic staining for HMGB1 in the syncytiotrophoblast and cytotrophoblasts (Fig. 1C).

Syncytial nuclear aggregates (SNAs) extruded from explants treated with aPL had strong cytoplasmic staining for HMGB1 (Fig. 1D). In contrast, syncytial nuclear aggregates (SNAs) shed from untreated or
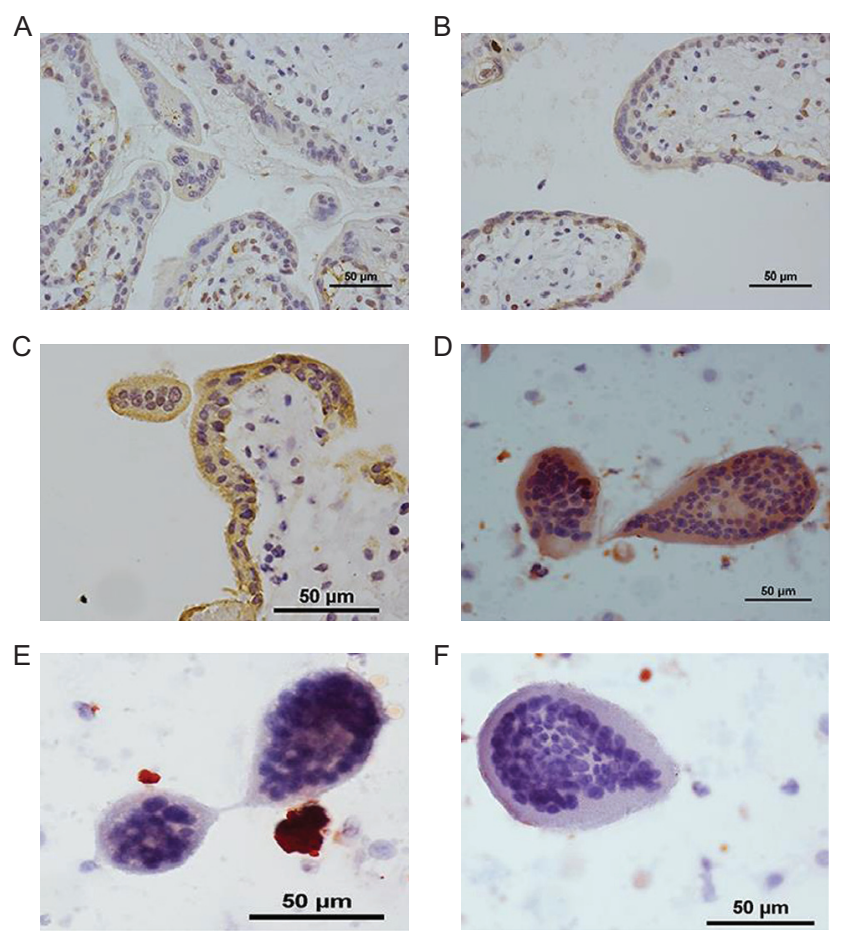

Figure 1 Photomicrographs demonstrating immunohistochemical staining for HMBG1 of first-trimester placental explants that had been (A) untreated, (B) treated with an isotype-matched control antibody or (C) had been treated with aPL for $24 \mathrm{~h}$. Photomicrographs demonstrating the immunohistochemical staining of HMGB1 in trophoblastic debris from placental explants that had been (D) treated with aPL or (E) treated with an isotype-matched control antibody (F) or from explants that were untreated for $24 \mathrm{~h}$. 


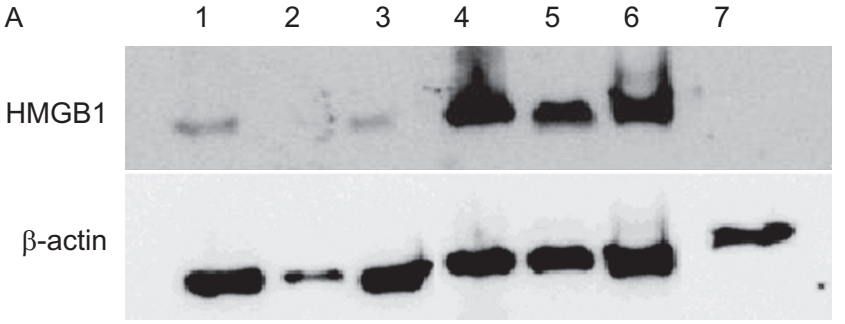

B

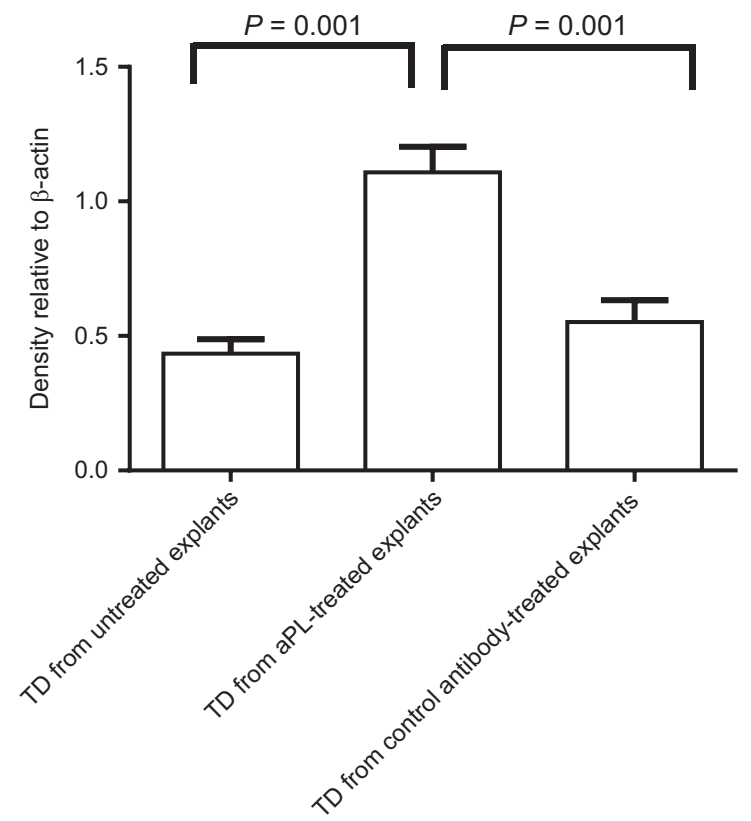

Figure 2 Representative western blot (A) demonstrating that the levels of HMGB1 were increased in trophoblastic debris (TD) from placental explants that had been treated with aPL $(n=16)$ (lanes 4-6) compared with trophoblastic debris from placental explants that had been treated for $24 \mathrm{~h}$ with isotype-matched control antibody $(n=6)$ (lane 7) or explants that were untreated $(n=16)$ (lanes $1-3)$.

(B) Semi-quantitative analysis showed that when normalised to levels of $\beta$-actin, the levels of HMGB1 were significantly increased in trophoblastic debris from placental explants that had been treated with aPL for $24 \mathrm{~h}$.

isotype-matched control antibody-treated explants did not stain for HMGB1 (Fig. 1E and F). The fact that the increase in levels of HMGB1 in trophoblastic debris (SNAs) from placental explants that had been treated with aPL was significant was confirmed by western blot (Fig. 2).

To confirm the increase in HMGB1 in the cytoplasm of the syncytiotrophoblast, we isolated a fraction enriched for syncytiotrophoblast cytoplasm from explants and confirmed a significant $(P<0.05)$ increase in cytoplasmic HMGB1 in response to treatment with aPL compared with an isotypematched control antibody (Fig. 3). Western blotting for the nuclear protein Lamin B1 confirmed that this fraction was enriched for cytoplasmic material (data not shown).

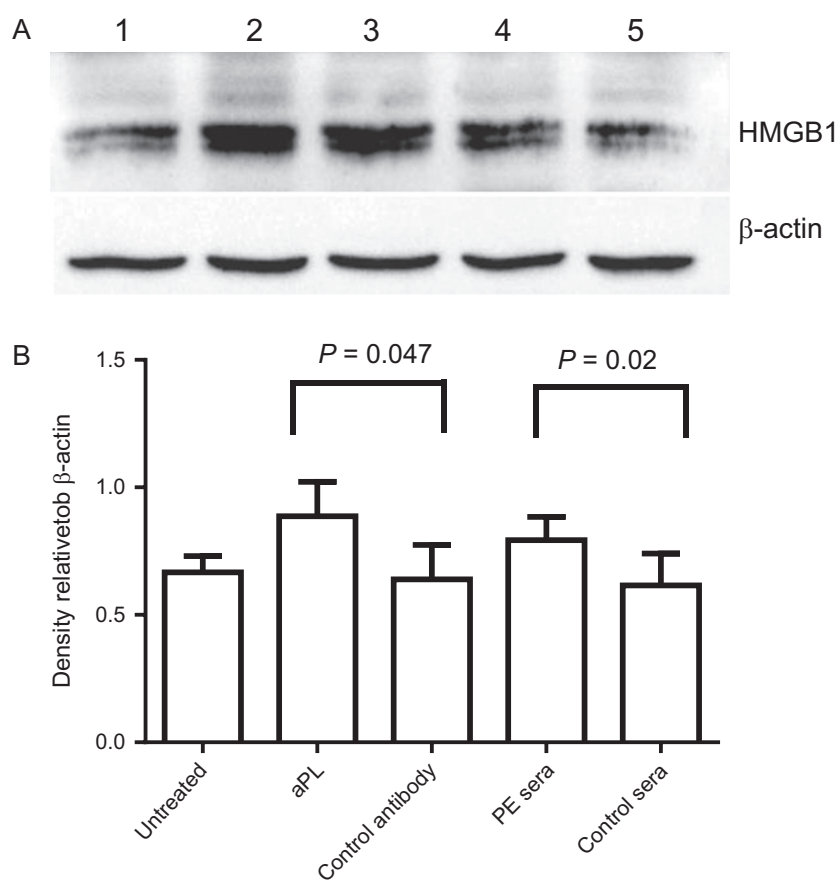

Figure 3 Representative western blot demonstrated that the levels of HMGB1 were increased in the cytoplasmic fraction of the syncytiotrophoblast from explants that had been treated with aPL (lane 2) or preeclamptic sera (lane 4) compared with untreated explants (lane 1) or explants that had been treated with isotypematched control antibody (lane 3) or normotensive control sera (lane 5) (A). Semi-quantitative analysis showed that when normalised to levels of $\beta$-actin, the levels of HMGB1 were significantly increased in explants that had been treated with aPL or preeclamptic sera (B).
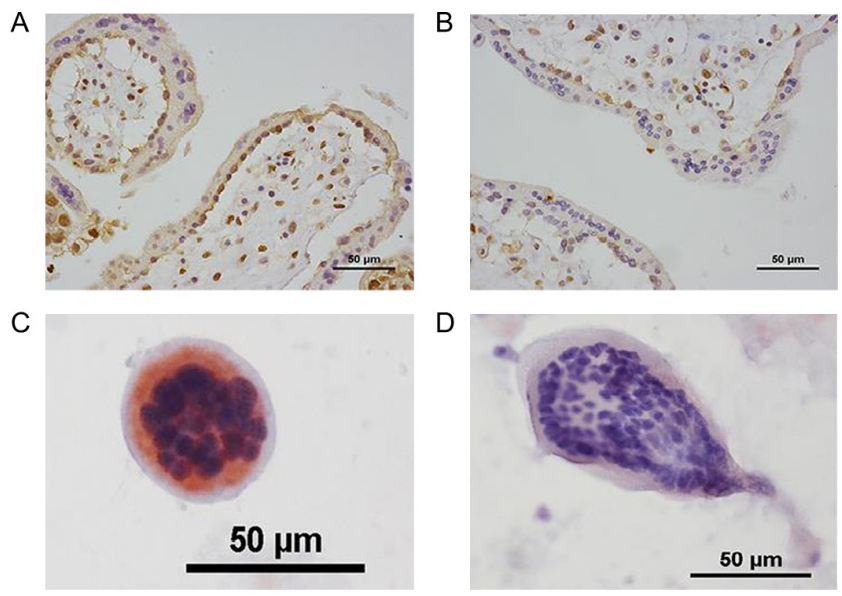

Figure 4 Representative photomicrographs from an immunohistochemical analysis of first trimester placental explants that had been treated with (A) $10 \%$ preeclamptic sera or (B) $10 \%$ normotensive control sera for $24 \mathrm{~h}$ or trophoblastic debris from placental explants that had been treated with (C) preeclamptic sera or (D) control normotensive sera for $24 \mathrm{~h}$. The samples were probed with an anti-HMGB1 antibody. 


\section{The expression of HMGB1 was increased by treating placental explants with preeclamptic sera}

Immunohistochemical and western blotting analyses indicated that the expression of HMGB1, particularly in the cytoplasm of the syncytiotrophoblast, was increased in placentae after treatment with preeclamptic sera (Figs 3 and 4) compared with explants treated with sera from normotensive pregnant women (Figs 3 and 4). Likewise, the level of HMGB1 in the cytoplasm of trophoblastic debris extruded from explants that had been treated with preeclamptic sera (Fig. 4C) was increased compared with debris from explants treated with sera from normotensive pregnant women (Fig. 4D).

\section{The level of HMGB1 in conditioned medium from placentae treated with aPL or preeclamptic sera was increased}

As our data mentioned previously showed that the expression of HMGB1 is increased in the cytoplasm of the syncytiotrophoblast as well as, in trophoblastic debris, we also quantified the levels of HMGB1 in conditioned medium from placental explants culture that had been treated with $\mathrm{aPL}$ or preeclamptic sera, as well as the relevant controls. The levels of HMGB1 were significantly increased in conditioned medium from explants that had been treated with aPL compared with those from explants that were untreated or treated with the isotype-matched control antibody (Fig. 5). However, there was no significant increase in the levels of HMGB1 in conditioned medium from explants that had been treated with preeclamptic compared with normotensive control sera (data not shown).

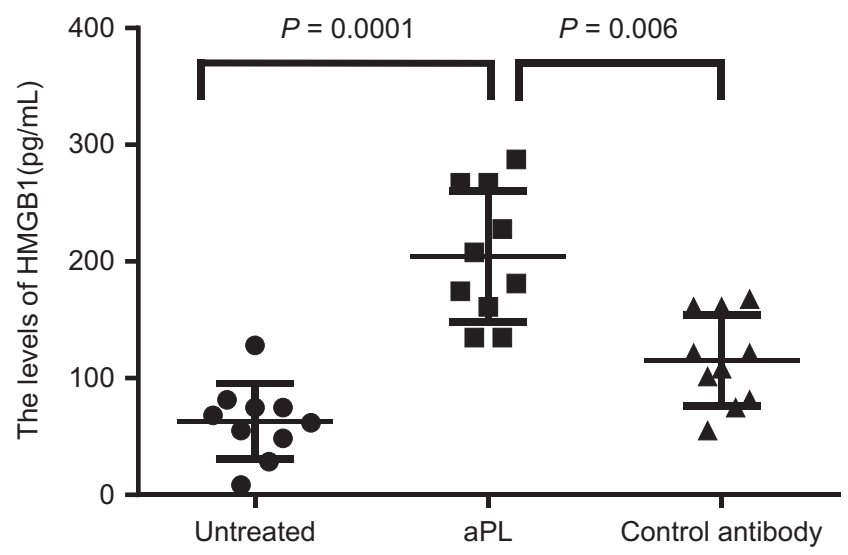

Figure 5 The levels of HMGB1 in conditioned medium from placental explants that had been treated with aPL for $24 \mathrm{~h}$ was significantly increased compared with untreated placental explants $(P=0.0001)$ or conditioned medium from explants that had been treated with an isotype-matched control antibody $(P=0.006)$ as measured by a commercial ELISA kit.

\section{HMGB1 induced endothelial cell activation}

Our previous studies showed that after exposure to trophoblastic debris extruded from placental explants treated with aPL or preeclamptic sera, endothelial cells became activated increasing their expression of cell-surface ICAM-1 and increasing monocyte adhesion (Chen et al. 2006, 2009a). To investigate whether increased levels of HMGB1 in trophoblastic debris could be one of the causes of this endothelial cell activation, we treated endothelial cells with
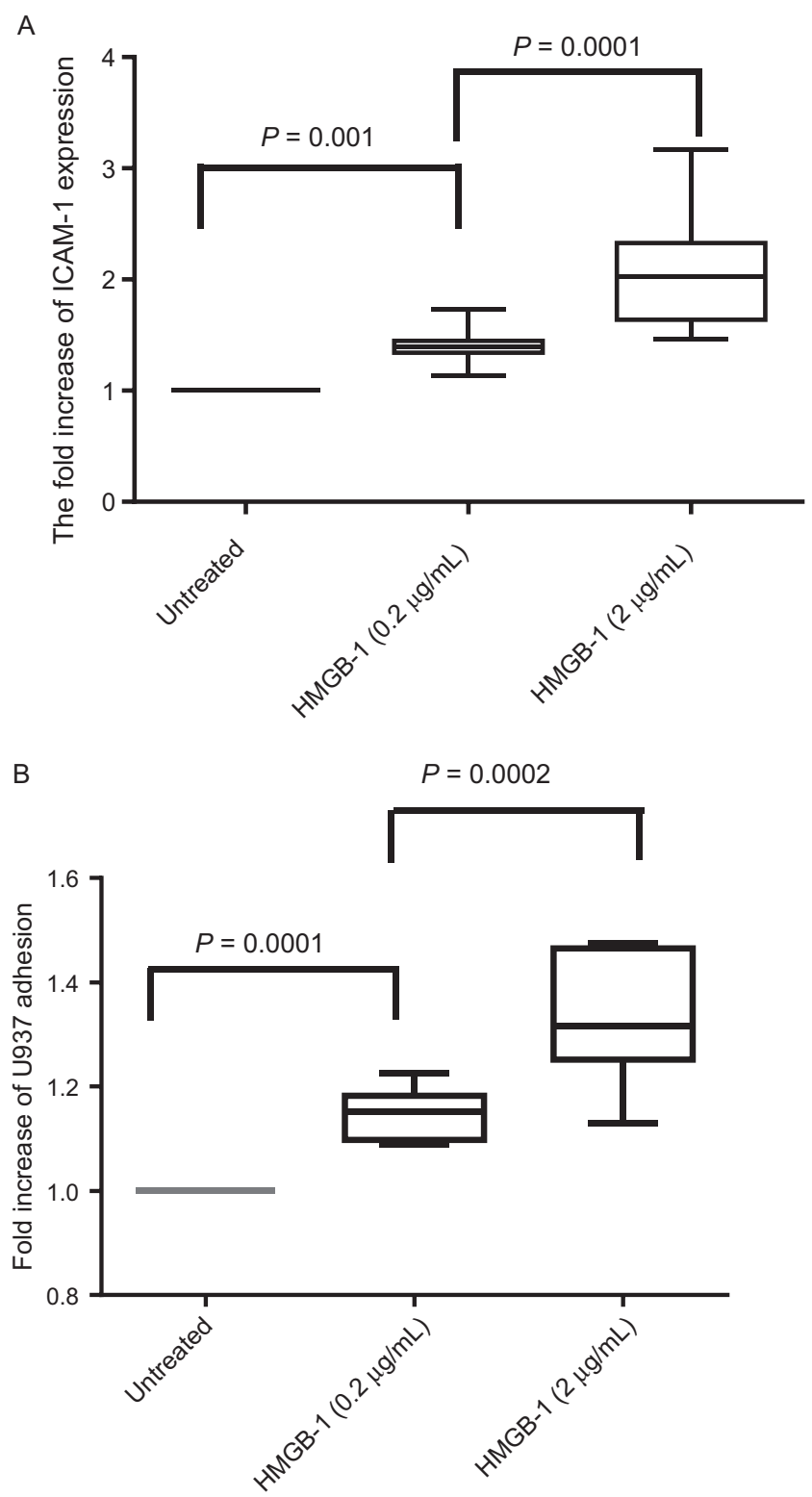

Figure 6 The level of, (A) expression of cell-surface ICAM-1 by monolayers of HMEC-1 cells determined by ELISA or (B) of U937 monocyte adhesion to HMEC-1 cells that were exposed to human recombinant HMGB1 (either $0.2 \mu \mathrm{g}$ or $2 \mu \mathrm{g}$ ) for $24 \mathrm{~h}$ was significantly increased ( $P=0.0001, \mathrm{ANOVA})$. Quadruplicate wells of endothelial cells were exposed to each treatment during each of the three separate experiments. Graphs show median and 5-95th percentiles. 

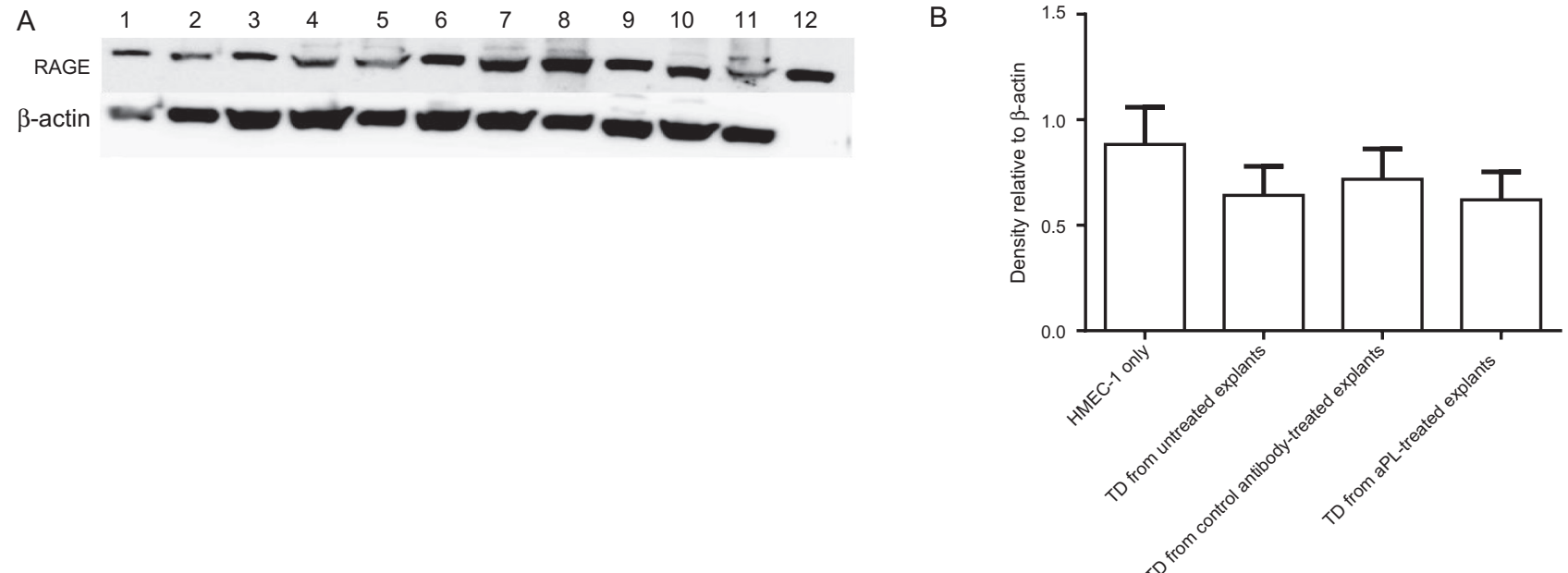

Figure 7 Representative western blots demonstrating (A) the levels of RAGE expressed by untreated HMEC-1s (lanes 1 and 2) or HMEC-1 cells exposed to trophoblastic debris (TD) from untreated placental explants (lanes 3-5) or HEMC-1 cells exposed to trophoblastic debris from placental explants that had been treated with an isotype-matched control antibody (lanes 6-8) for $24 \mathrm{~h}$ or HEMC-1s exposed to trophoblastic debris from placental explants that had been treated with aPL for $24 \mathrm{~h}$ (lanes 9-11) or normal plasma (positive control, lane 12). Levels of RAGE in the samples were normalised to $\beta$-actin (B), and there was no statistical difference among the groups. Western blots were repeated three times.

increasing concentrations of recombinant HMGB1, which resulted in a concentration-dependent, significant increase in cell-surface ICAM-1 expression and adhesion of $U 937$ monocytes to the endothelial cells (Fig. 6, $P=0.0001$ ANOVA).

\section{RAGE was expressed in endothelial cells}

HMGB1 instigates many of its proinflammatory actions via upregulation of its receptor RAGE. Western blotting identified that RAGE was expressed by the HMEC1 endothelial cells, but RAGE was not upregulated by the endothelial cells in response to trophoblastic debris from explants that had been treated with aPL (Fig. 7). We then investigated whether blocking RAGE could block the effect of trophoblastic debris extruded from placental explants treated with aPL on endothelial cells. The increased levels of ICAM-1 induced by trophoblastic debris extruded from placental explants treated with aPL were partially reversed by treating the endothelial cells with a RAGE-neutralising antibody (Fig. 8).

\section{Discussion}

During normal pregnancy, trophoblastic debris is produced and shed into the maternal blood in large quantities where it is cleared partly by maternal endothelial cells. In normal pregnancies, the trophoblastic debris is 'safe' and has been shown to prevent endothelial cells from becoming activated (Chen et al. 2012a). In contrast, when placentae are exposed to preeclamptic sera or to aPL (a major risk factor for preeclampsia), the resultant trophoblastic debris is dangerous/toxic and activates endothelial cells. It is not clear why exposing placental explants to
aPL or preeclamptic serum induces the trophoblastic debris shed from the explants to become dangerous and activate endothelial cells. However, previous studies have shown that the danger signal, HMGB1, is present in the syncytiotrophoblast of preeclamptic pregnancies at increased levels (Zhu et al. 2015).

In this study, we have demonstrated that one change which occurs in placentae exposed to aPL or preeclamptic sera is an increase in the amount of cytoplasmic HMGB1 in the syncytiotrophoblast. This increase in cytoplasmic HMGB1 is specific to the syncytiotrophoblast (from which trophoblastic debris is derived) because a similar increase in HMGB1 did not occur in the mesenchymal cells, which also express basal levels of HMGB1, primarily in their nuclei. HMGB1 was originally described as a DNAbinding nuclear protein, and it is expressed by many cells, including trophoblasts (Wang et al. 2011). However, under conditions of stress or cell death, HMGB1 can translocate to the cytoplasm where it can regulate processes such as autophagy and apoptosis (Tsung et al. 2014). HMGB1 can also be secreted into the extracellular environment, but like some other proinflammatory proteins (e.g. IL-1 $\beta$ ), HMGB1 lacks a leader peptide and thus is not secreted through the cytoplasmic membrane via the classical protein secretion pathway. Instead in activated macrophages/ monocytes, it is secreted via endolysosomal vesicles that are found in the extracellular environment as microvesicles that have budded from the plasma membrane (Gardella et al. 2002, Chen et al. 2016a). In this study, we quantified the level of expression of HMGB1 in cytoplasm, and we further found that placental explants that were treated with $\mathrm{aPL}$ or preeclamptic sera increased the levels of HMGB1 in the 


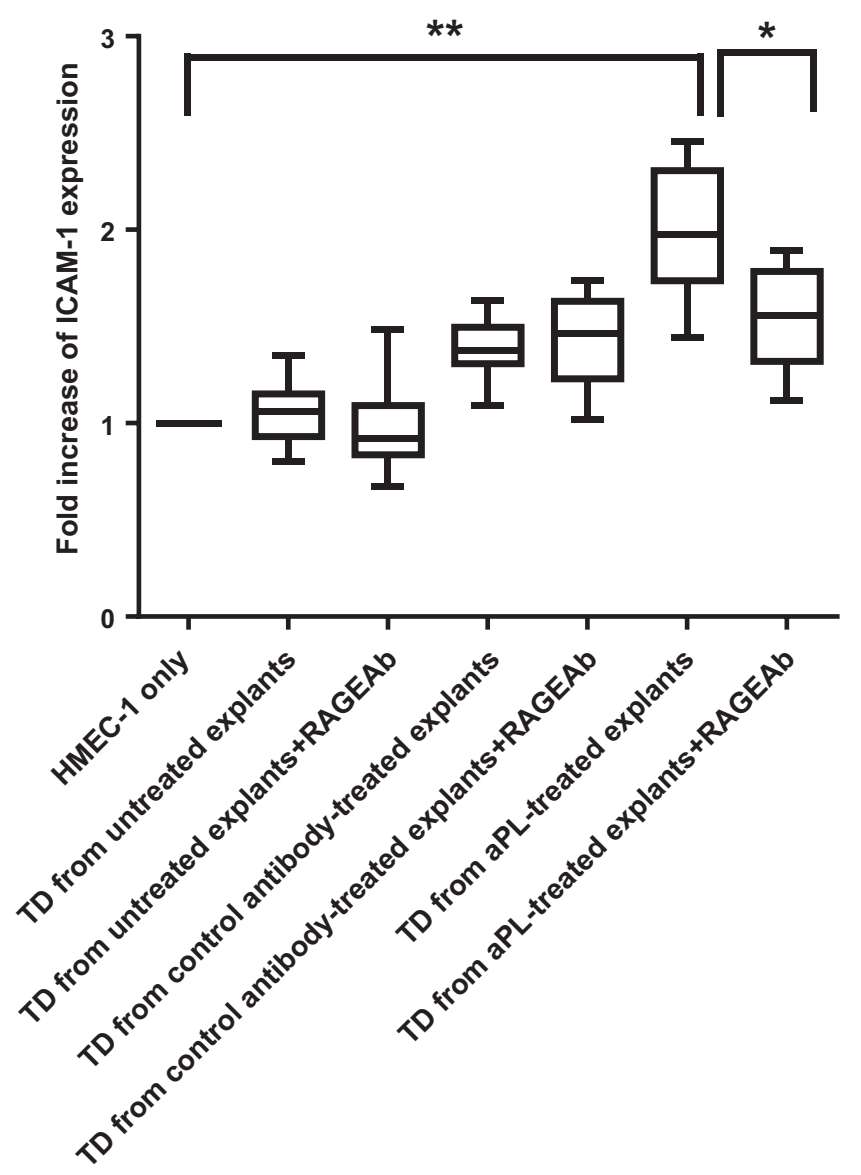

Figure 8 Cell-surface ELISA was used to determine the levels of expression of cell-surface ICAM-1 by monolayers of HMEC-1 that were exposed to trophoblastic debris (TD) extruded from placental explants that were untreated or treated with an isotype-matched control antibody or with aPL for $24 \mathrm{~h}$ in the presence or absence of a RAGE neutralising antibody (RAGEAb). Trophoblastic debris from aPL-treated placental explants significantly increased the ICAM-1 expression (** $P=0.0001)$; however, this increased ICAM-1 expression was significantly reduced by adding the RAGE-neutralising antibody $\left({ }^{*} P=0.0001\right)$. Quadruplicate wells of endothelial cells were exposed to each treatment during each of three separate experiments. Graphs show median and 5-95th percentiles. (RAGEAb, RAGE neutralising antibody; TD, trophoblastic debris.)

cytoplasm of syncytiotrophoblast or cytotrophoblast. This suggests that these treatments (aPL or preeclamptic sera) are in some way stressing the syncytiotrophoblast resulting in the translocation of HMGB1 from the nucleus to the cytoplasm. Indeed, we have previously shown that aPL can enter the syncytiotrophoblast and disrupt mitochondrial function, which is highly likely to induce stress in the syncytiotrophoblast (Viall et al. 2013). Although the exact origin of trophoblastic debris is unclear, multinucleated syncytial nuclear aggregates, which are part of the debris, are membrane enclosed structures that possibly bud from the syncytiotrophoblast membrane and thus inclusion of HMGB1 in SNAs may be equivalent to the secretion of HMGB1 via microvesicles from mononuclear cells. When released from the cytoplasm of cells, HMGB1 has been shown to act as a danger-associated molecular pattern (DAMP) or 'danger signal'. As cytoplasmic HMBG1 has the potential to be a danger signal, the apparent specific increase in cytoplasmic HMGB1 suggests that it is likely to be able to act as a danger signal if released from the syncytiotrophoblast. Although we did not quantify it, there was also an apparent general increase in the expression of HMGB1 in response to treatment of the explants with aPL. This increase may simply reflect an attempt to correct the loss of HMBG1 from the nucleus where it is the most abundant nonhistone DNA-binding protein and performs important functions such as regulating DNA structure, replication and repair and is a transcription cofactor (Tsung et al. 2014). Our demonstration that debris released from aPL or preeclamptic sera-treated placentae contained increased amounts of cytoplasmic HMGB1 suggests that this danger signal in trophoblastic debris has the potential to activate endothelial cells that phagocytose trophoblastic debris. Our data also showed that extracellular levels of HMGB1 were significantly increased by treatment with aPL. In vivo, this secreted HMGB1 would be released into the maternal blood during pregnancy and may, along with the HMGB1 in trophoblastic debris, contribute to maternal endothelial cell activation in women with aPL. Therefore, to test this possibility, we confirmed that stimulation of endothelial cells with human recombinant HMGB1 resulted in the activation of endothelial cells as shown by an increase in the expression of ICAM-1 and U937 adhesion to endothelial cells. Consistent with other studies (Lee et al. 2015), our result suggests that extracellular HMGB1 acts as a danger signal to mediate endothelial cell activation and inflammation.

HMGB1 has several potential receptors (including TLRs 2, 4 and 9) (reviewed in Harris et al. 2012). RAGE is expressed by endothelial cells and is thought to be the key receptor in HMGB1-mediated endothelial cell activation (Luo et al. 2013). RAGE is a cell-surface receptor of the immunoglobulin superfamily expressed in many cells including endothelial cells. Our data confirm that the RAGE is expressed by endothelial cells and show that inhibiting RAGE with a function-blocking antibody partly reduced the activation of endothelial cells in response to trophoblastic debris from aPLtreated explants. This suggests that the increased levels of HMGB1 in this debris are partially responsible for inducing endothelial cell activation and that this signalling is, at least in part, via RAGE.

Other studies have shown that the expression of RAGE is increased when its ligands accumulate (Schmidt et al. 2001) or when endothelial cells are exposed to HMGB1 (Huang et al. 2012). However, we did not see a similar increase in the level of RAGE by endothelial cells exposed to trophoblastic debris from aPL or preeclamptic sera-treated explants. We do not know the reason for 
this difference. However, this discrepancy may relate to the manner in which HMGB1 in trophoblast debris is exposed to the endothelial cell RAGE. For example, we do not know whether the HMGB1 from trophoblastic debris is released before phagocytosis of the debris by endothelial cells, thus being available to bind to cellsurface RAGE or at some later stage during/after the phagocytosis of the debris by the endothelial cells.

Although this study specifically focused on the role of HMGB1 in dangerous trophoblastic debris, it is unlikely that a single molecule is the only signal that renders trophoblastic debris dangerous. We have previously reported that trophoblastic debris from preeclamptic placentae contains elevated levels of the proinflammatory cytokine IL-1 $\beta$, which is in part responsible for activating endothelial cells. We believe that it is likely that debris from preeclamptic placentae contains several, if not numerous, molecules that together contribute to the dangerous nature of such debris, and which contribute to the pathogenesis of preeclampsia.

In conclusion, our study clearly demonstrates that treating normal placental explants with either antiphospholipid antibodies or preeclamptic sera increases the level of the danger signal HMGB1 in the cytoplasm of the syncytiotrophoblast. Moreover, the trophoblastic debris extruded from these treated explants also contains increased amounts of cytoplasmic HMGB1, which seems able to interact with RAGE (and possibly other receptors) on endothelial cells. This interaction in part contributes to the activation of these endothelial cells in response to this debris. Thus HMGB1, contained in trophoblastic debris, is likely to be one of the toxic factors released from the placenta that contribute to the pathogenesis of preeclampsia.

\section{Declaration of interest}

The authors declare that there is no conflict of interest that could be perceived as prejudicing the impartiality of the research reported.

\section{Funding}

This study was supported by grants from Auckland Medical Research Foundation (AMRF). Drs Jun Shao and Mingzhi Zhao were supported by a grant from the Hospital of Obstetrics \& Gynaecology, Fudan University, Peoples' Republic of China. J W is the recipient of Chinese Scholarship Council, Peoples' Republic of China.

\section{Acknowledgements}

The authors would like to thank all the staff at Epsom Day Unit, Auckland City Hospital and Auckland Medical Aid Centre for their help in collecting samples for this study. The authors also thank the women who donated blood and tissue for this study.

\section{References}

Abumaree M, Stone P \& Chamley L 2006 An in vitro model of human placental trophoblast deportation/shedding. Molecular Human Reproduction 12 687. (doi:10.1093/molehr/gal073)

Ades EW, Candal FJ, Swerlick RA, George VG, Summers S, Bosse DC \& Lawley TJ 1992 HMEC-1: establishment of an immortalized human microvascular endothelial cell line. Journal of Investigative Dermatology 99 683-690. (doi:10.1111/1523-1747.ep12613748)

Attwood HD \& Park WW 1961 Embolism to the lungs by trophoblast. Journal of Obstetrics and Gynaecology of the British Commonwealth 68 611-617. (doi:10.1111/j.1471-0528.1961.tb02778.x)

Bianchi ME \& Manfredi AA 2007 High-mobility group box 1 (HMGB1) protein at the crossroads between innate and adaptive immunity. Immunological Reviews 220 35-46. (doi:10.1111/j.1600065X.2007.00574.X)

Campana L, Bosurgi L \& Rovere-Querini P 2008 HMGB1: a two-headed signal regulating tumor progression and immunity. Current Opinion in Immunology 20 518-523. (doi:10.1016/j.coi.2008.04.012)

Chamley LW, Konarkowska B, Duncalf AM, Mitchell MD \& Johnson PM 2001 Is interleukin-3 important in antiphospholipid antibody-mediated pregnancy failure? Fertility and Sterility 76 700-706. (doi:10.1016/ S0015-0282(01)01984-7)

Chen Q, Ding JX, Liu B, Stone P, Feng YJ \& Chamley L 2010 Spreading endothelial cell dysfunction in response to necrotic trophoblasts. Soluble factors released from endothelial cells that have phagocytosed necrotic shed trophoblasts reduce the proliferation of additional endothelial cells. Placenta 31 976-981. (doi:10.1016/j.placenta.2010.08.013)

Chen Q, Guo F, Jin HY, Lau S, Stone P \& Chamley L 2012a Phagocytosis of apoptotic trophoblastic debris protects endothelial cells against activation. Placenta 33 548-553. (doi:10.1016/j.placenta.2012.03.007)

Chen Q, Guo F, Liu S, Xiao J, Wang C, Snowise S, Stone PR \& Chamley LW $2012 b$ Calcium channel blockers prevent endothelial cell activation in response to necrotic trophoblast debris: possible relevance to preeclampsia. Cardiovascular Research 96 484-493. (doi:10.1093/cvr) cvs279)

Chen Q, Stone P, Ching LM \& Chamley L 2009a A role for interleukin-6 in spreading endothelial cell activation after phagocytosis of necrotic trophoblastic material: implications for the pathogenesis of preeclampsia. Journal of Pathology 217 122-130. (doi:10.1002/path.2425)

Chen Q, Viall C, Kang Y, Liu B, Stone P \& Chamley L 2009b Antiphospholipid antibodies increase non-apoptotic trophoblast shedding: a contribution to the pathogenesis of pre-eclampsia in affected women? Placenta 30 767-773. (doi:10.1016/j.placenta.2009.06.008)

Chen Q, Stone PR, McCowan LM \& Chamley LW 2006 Phagocytosis of necrotic but not apoptotic trophoblasts induces endothelial cell activation. Hypertension 47 116-121. (doi:10.1161/01. HYP.0000196731.56062.7c)

Chen Y, Li G, Liu Y, Werth VP, Williams KJ \& Liu ML 2016a Translocation of endogenous danger signal HMGB1 from nucleus to membrane microvesicles in macrophages. Journal of Cellular Physiology 231 2319-2326. (doi:10.1002/jcp.25352)

Chen Q, Yin YX, Wei J, Tong M, Shen F, Zhao M \& Chamley L 2016 b Increased expression of high mobility group box 1 (HMGB1) in the cytoplasm of placental syncytiotrophoblast from preeclamptic placentae. Cytokine 85 30-36. (doi:10.1016/j.cyto.2016.06.001)

Chen Q, Zhang Y, Tong M, Wu M, Snowise S, Stone P \& Chamley LW 2013 Pre-treatment with calcium prevents endothelial cell activation induced by multiple activators, necrotic trophoblastic debris or IL-6 or preeclamptic sera: possible relevance to the pathogenesis of preeclampsia. Placenta 34 1196-1201. (doi:10.1016/j.placenta.2013.09.014)

Covone AE, Mutton D, Johnson PM \& Adinolfi M 1984 Trophoblast cells in peripheral blood from pregnant women. Lancet 2 841-843. (doi:10.1016/S0140-6736(84)90875-4)

Douglas GW, Thomas L, Carr M, Cullen NM \& Morris R 1959 Trophoblast in the circulating blood during pregnancy. American Journal of Obstetrics and Gynecology 78 960-973.

Duckitt K \& Harrington D 2005 Risk factors for pre-eclampsia at antenatal booking: systematic review of controlled studies. BMJ 330565. (doi:10.1136/bmj.38380.674340.E0)

Gardella S, Andrei C, Ferrera D, Lotti LV, Torrisi MR, Bianchi ME \& Rubartelli A 2002 The nuclear protein HMGB1 is secreted by monocytes 
via a non-classical, vesicle-mediated secretory pathway. EMBO Reports 3 995-1001. (doi:10.1093/embo-reports/kvf198)

Harris HE, Andersson U \& Pisetsky DS 2012 HMGB1: a multifunctional alarmin driving autoimmune and inflammatory disease. Nature Reviews Rheumatology 8 195-202. (doi:10.1038/nrrheum.2011.222)

Hori O, Brett J, Slattery T, Cao R, Zhang J, Chen JX, Nagashima M, Lundh ER, Vijay S, Nitecki D et al. 1995 The receptor for advanced glycation end products (RAGE) is a cellular binding site for amphoterin. Mediation of neurite outgrowth and co-expression of rage and amphoterin in the developing nervous system. Journal of Biological Chemistry 270 25752-25761. (doi:10.1074/jbc.270.43.25752)

Huang W, Liu Y, Li L, Zhang R, Liu W, Wu J, Mao E \& Tang Y 2012 HMGB1 increases permeability of the endothelial cell monolayer via RAGE and Src family tyrosine kinase pathways. Inflammation 35 350-362. (doi:10.1007/s10753-011-9325-5)

Huppertz B, Kingdom J, Caniggia I, Desoye G, Black S, Korr H \& Kaufmann P 2003 Hypoxia favours necrotic versus apoptotic shedding of placental syncytiotrophoblast into the maternal circulation. Placenta 24 181-190. (doi:10.1053/plac.2002.0903)

Hypertension in Pregnancy 2013 American College of Obstetricians and Gynecologists. Women's Health Care Physician.

Knight M, Redman CW, Linton EA \& Sargent IL 1998 Shedding of syncytiotrophoblast microvilli into the maternal circulation in preeclamptic pregnancies. British Journal of Obstetrics and Gynaecology 105 632-640. (doi:10.1111/j.1471-0528.1998.tb10178.x)

Lau SY, Guild SJ, Barrett CJ, Chen Q, McCowan L, Jordan V \& Chamley LW 2013 Tumor necrosis factor-alpha, interleukin-6, and interleukin-10 levels are altered in preeclampsia: a systematic review and metaanalysis. American Journal of Reproductive Immunology 70 412-427. (doi:10.1111/aji.12138)

Lee W, Ku SK, Na DH \& Bae JS 2015 Anti-inflammatory effects of lysozyme against HMGB1 in human endothelial cells and in mice. Inflammation 38 1911-1924. (doi:10.1007/s10753-015-0171-8)

Lotze MT \& Tracey KJ 2005 High-mobility group box 1 protein (HMGB1): nuclear weapon in the immune arsenal. Nature Reviews Immunology $\mathbf{5}$ 331-342. (doi:10.1038/nri1594)

Luo Y, Li SJ, Yang J, Qiu YZ \& Chen FP 2013 HMGB1 induces an inflammatory response in endothelial cells via the RAGE-dependent endoplasmic reticulum stress pathway. Biochemical and Biophysical Research Communications 438 732-738. (doi:10.1016/j.bbrc.2013.07.098)

Noris M, Perico N \& Remuzzi G 2005 Mechanisms of disease: preeclampsia. Nature Clinical Practice Nephrology 1 98-114. (doi:10.1038/ ncpneph0035)

Pantham P, Viall CA, Chen Q, Kleffmann T, Print CG \& Chamley LW 2015 Antiphospholipid antibodies bind syncytiotrophoblast mitochondria and alter the proteome of extruded syncytial nuclear aggregates. Placenta 36 1463-1473. (doi:10.1016/j.placenta.2015.10.006)

Park JS, Gamboni-Robertson F, He Q, Svetkauskaite D, Kim JY, Strassheim D, Sohn JW, Yamada S, Maruyama I, Banerjee A et al. 2006 High mobility group box 1 protein interacts with multiple Tolllike receptors. American Journal of Physiology: Cell Physiology 290 C917-C924. (doi:10.1152/ajpcell.00401.2005)

Redman CW \& Sargent IL 2010 Immunology of pre-eclampsia. American Journal of Reproductive Immunology 63 534-543. (doi:10.1111/j.16000897.2010.00831.x)

Schmidt AM, Yan SD, Yan SF \& Stern DM 2001 The multiligand receptor RAGE as a progression factor amplifying immune and inflammatory responses. Journal of Clinical Investigation 108 949-955. (doi:10.1172/ JCI14002)

Shen F, Wei J, Snowise S, DeSousa J, Stone P, Viall C, Chen Q \& Chamley L 2014 Trophoblast debris extruded from preeclamptic placentae activates endothelial cells: a mechanism by which the placenta communicates with the maternal endothelium. Placenta 35 839-847. (doi:10.1016/j. placenta.2014.07.009)

Sibai B, Dekker G \& Kupferminc M 2005 Pre-eclampsia. Lancet 365 785-799. (doi:10.1016/S0140-6736(05)71003-5)

Tsung A, Tohme S \& Billiar TR 2014 High-mobility group box-1 in sterile inflammation. Journal of Internal Medicine 276 425-443. (doi:10.1111/ joim.12276)

Viall CA, Chen Q, Liu B, Hickey A, Snowise S, Salmon JE, Stone PR \& Chamley LW 2013 Antiphospholipid antibodies internalised by human syncytiotrophoblast cause aberrant cell death and the release of necrotic trophoblast debris. Journal of Autoimmunity 47 45-57. (doi:10.1016/j. jaut.2013.08.005)

Wang B, Koga K, Osuga Y, Hirata T, Saito A, Yoshino O, Hirota Y, Harada M, Takemura Y, Fujii T et al. 2011 High mobility group box 1 (HMGB1) levels in the placenta and in serum in preeclampsia. Journal of Reproductive Immunology 66 143-148. (doi:10.1111/j.16000897.2010.00975.x)

Zhu L, Zhang Z, Zhang L, Shi Y, Qi J, Chang A, Gao J, Feng Y \& Yang X 2015 HMGB1-RAGE signaling pathway in severe preeclampsia. Placenta $\mathbf{3 6}$ 1148-1152. (doi:10.1016/j.placenta.2015.08.006)

Received 22 February 2016

First decision 5 April 2016

Revised manuscript received 3 August 2016

Accepted 22 September 2016 